\title{
Preparation and structural characterization of sodium polyphosphate coacervate as a precursor for optical materials
}

\author{
D.F. Franco ${ }^{\text {a, * }}$, H.S. Barud ${ }^{\text {b }}$, S. Santagneli ${ }^{\text {a }}$, R.S. Lamarca ${ }^{\text {d }}$, Bruno F. Santos ${ }^{\text {e, M.A.P. Silva }}{ }^{\text {, }}$, \\ L.F.C. de Oliveira ${ }^{\text {c }}$, S.J.L. Ribeiro ${ }^{a}$, M. Nalin ${ }^{a}$ \\ a Institute of Chemistry - São Paulo State University - Unesp, P.O. Box 355, Araraquara, SP, 14801-970, Brazil \\ ${ }^{\mathrm{b}}$ Laboratório de Biopolímeros e Biomateriais (BioPolMat) - Centro Universitário de Araraquara (UNIARA), Araraquara, SP, Brazil \\ ${ }^{\mathrm{c}}$ Núcleo de Espectroscopia e Estrutura Molecular - NEEM, Departamento de Química, Instituto de Ciências Exatas, Universidade Federal de Juiz de Fora, \\ 36036-900, Juiz de Fora, MG, Brazil \\ ${ }^{\mathrm{d}}$ NUPIS (Núcleo de Pesquisa em Instrumentação e Separações Analíticas), Departamento de Química, Instituto de Ciências Exatas, Universidade Federal de \\ Juiz de Fora, 36036-900, Juiz de Fora, MG, Brazil \\ e School of Pharmaceutical Sciences, São Paulo State University - UNESP, Araraquara, SP, 14801-902, Brazil
}

\section{H I G H L I G H T S}

- Sodium Coacervates Polyphosphates (NaPC) were prepared.

- Methanol reduces the dielectric constant and it leads to coacervation process.

- NaPC can be used as soft Glass-Materials precursors.
G R A P H I C A L A B S T R A C T

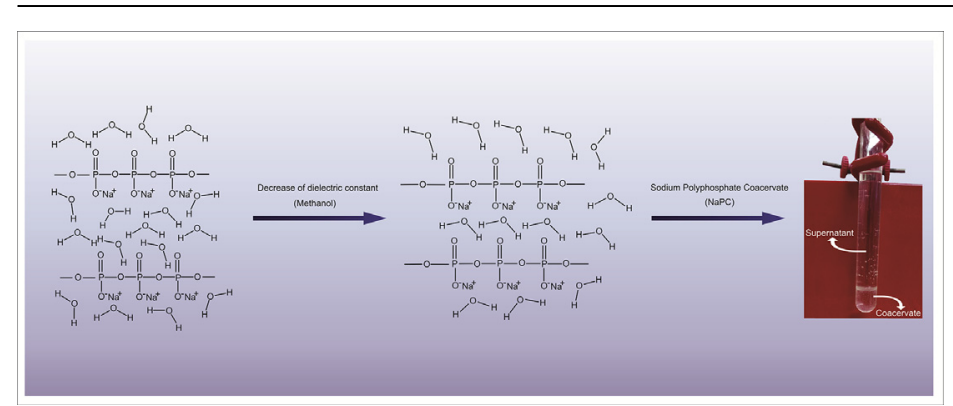

\section{A R T I C L E I N F O}

\section{Article history:}

Received 5 February 2016

Received in revised form

16 May 2016

Accepted 21 May 2016

Available online 27 May 2016

\section{Keywords:}

Coacervates

Glasses

Optical properties

Nuclear magnetic resonance (NMR)

\begin{abstract}
A B S T R A C T
This paper describes the preparation of Sodium Polyphosphates Coacervates (NaPC) through the direct addition of different methanol molar fractions to a sodium polyphosphate solution. The presence of methanol reduces the dielectric constant medium and it promotes the coacervate formation. Rheology measurement shows that the NaPC viscosity is dependent on a methanol molar fraction, which is caused by the dielectric constant reduction of the water-methanol mixture. Raman spectra for the lyophilized NaPC show that the symmetric $\mathrm{P}-\mathrm{O}_{\mathrm{t}}$ stretching mode is more sensitive than the symmetric $\mathrm{P}-\mathrm{O}_{\mathrm{b}}$ stretching mode, due to the approach of the adjacent polyphosphate chains. The ${ }^{31} \mathrm{P}$ NMR spectra also confirm a decrease in the $\mathrm{Q}^{2} / \mathrm{Q}^{1}$ ratio because of the methanol content. Besides, we have obtained transparent soft Glass-Materials to melt NaPC at $800{ }^{\circ} \mathrm{C}$.
\end{abstract}

(c) 2016 Elsevier B.V. All rights reserved.

\footnotetext{
* Corresponding author.

E-mail address: fazafranco@yahoo.com.br (D.F. Franco).
}

\section{Introduction}

Polyphosphate Coacervates (PC) are colloidal systems obtained by polyphosphate solutions destabilization through the addition of electrolytes or solvents solutions featuring a lower dielectric 
constant than water [1]. "Coacervates", is a word derived from Latin, in which "co" (together) and "acerv" (a heap) [2-4]. They are formed by the interaction between aqueous sodium polyphosphates solutions and different cations, including $\mathrm{Ca}^{2+}, \mathrm{Mn}^{2+}$, $\mathrm{Co}^{2+}, \mathrm{Ni}^{2+}, \mathrm{Fe}^{3+} \mathrm{Eu}^{3+}$ and $\mathrm{Al}^{3+}$, and lead to a separation of phases [5-10]. The most viscous phase, richer in colloids, is called coacervate, while the less viscous is called supernatant [1]. The interaction between the metaphosphate chains and the metal ions $\mathrm{Ca}^{2+}$ and $\mathrm{Eu}^{3+}$ has been studied in water through $\mathrm{Eu}^{3+}$ luminescence, FTIR and ${ }^{31}$ P NMR spectroscopy [11]. Two main families of sites can be identified for the metal ions in the aqueous polyphosphate colloidal system: (1) cagelike sites provided by polyphosphate chains and (2) a family which emerges followed by the saturation of cagelike sites. The occupation of this second family leads to supramolecular interactions between polyphosphate chains and a consequent destabilization of the colloidal system [11].

The coacervation process may be also induced by the addition of a low molecular weight solvent $[2,4]$. According to the method proposed by Umegaki et al. it is necessary the adding of $10 \%$ in volume of methanol in the solution to obtain the liquid-liquid phase separation and formation of the coacervates [1]. Willots et al. studied the coacervation process as an alternative route for glasses preparation. He highlights the use of ethanol as a solvent for the phase separation process [12]. Silva et al. prepared a great amount of transparent amorphous materials using the coacervation process of sodium polyphosphate or Graham Salt containing $\mathrm{Ni}^{2+}$ and $\mathrm{Co}^{2+}$ applying methanol to reduce the dielectric constant of the mixture $[5,13]$. The proposal and understanding of the coacervation process on the $\mathrm{Co}^{2+}$ and $\mathrm{Ni}^{2+}$ coacervates using $10 \%$ of methanol was also studied through X-ray Absorption Spectroscopy (EXAFS analysis) and Raman Spectroscopy [14]. Concerning the applications, coacervates have a great potential for bone substitution $[15,16]$, incorporation of organic compounds [17], drug delivery systems [18], biomedical applications [19], optical devices [20] and absorption of heavy metal ions [21].

Saegusa et al. studied the effect of the addition of three different alcohols in the preparation of lipid vesicles using the coacervation process [22]. In this study, Saegusa et al. prepared lipid vesicles (liposomes) using the coacervation process by the addition of methanol, ethanol and 1-propanol in the phospholipid water system to determine the optimum conditions for the formation of coacervation. Umegaki and Kanazawa researched the viscosities of magnesium and calcium highpolyphosphates coacervates in the range of $20^{\circ} \mathrm{C}-90^{\circ} \mathrm{C}$ without addition of ethanol [6]. The results showed that magnesium and calcium highpolyphosphates coacervates presented Newtonian behavior in different temperatures. The addition of water to magnesium highpolyphosphates decreased the viscosity while no degradation of the polyphosphate chains in solution was observed.

Momeni and Filiaggi studied the rheological properties of the polyphosphate coacervates, which demonstrated themselves as Newtonian liquids. Their low shear rates and viscosity are directly related to the chain length of the sodium polyphosphate and divalent cation type $\left(\mathrm{Ca}^{2+}, \mathrm{Sr}^{2+}\right.$ or $\left.\mathrm{Ca}^{2+}\right)$ for the material preparation. The study shows a small fraction exchange of $\mathrm{Ca}^{2+}$ for $\mathrm{Sr}^{2+}$ or $\mathrm{Ba}^{2+}$ allowing the obtaintion of more elastic coacervates with higher viscosity than that having only calcium coacervates [23].

In this paper, we studied the influence of different methanol molar fractions on the coacervation process of a sodium polyphosphate solution, without the presence of di- or trivalent metallic ions. Furthermore, a glass derived from sodium coacervate was prepared in order to demonstrate its potential as a new precursor for optical materials. We studied the structural and spectroscopic properties of the sodium coacervates by means of rheological measurements, scanning electronic microscopy (SEM), energy dispersive X-Ray spectroscopy (EDS), Raman spectroscopy,

${ }^{31} \mathrm{P}$ solid-state nuclear magnetic resonance (NMR), X-ray diffraction and differential scanning calorimeter (DSC).

\section{Experimental procedure}

\subsection{Coacervate preparation}

Sodium polyphosphate coacervates, NaPC, were prepared according to the methodology proposed earlier by Umegaki et al. [1]. Different molar fractions $(x=4.25 ; 8.17 ; 11.8 ; 15.1 ; 18.2 ; 23.7$ and $30.8 \%$ ) of methanol (Synth P.A) were slowly added to a $4 \mathrm{~mol} \mathrm{~L}^{-1}$ $\mathrm{Na}\left(\mathrm{PO}_{3}\right)_{\mathrm{n}}$ solution (Merck), under constant stirring. Samples were renamed as described in Table 1

\subsection{Coacervate characterization}

The viscosity behavior of the coacervates was studied by rheological measurements using TA instruments AR2000 rheometer at $25{ }^{\circ} \mathrm{C}$. For each sample, flow curves were measured at the increasing shear rate from 0.1 to $100 \mathrm{~s}^{-1}$. The flow curves were fitted by Power's law $\left(\sigma=\mathrm{k} \mathrm{g}^{\mathrm{n}}\right)$ to obtain flow behavior $(\mathrm{n})$; consistency index $(\mathrm{k})$; and coefficient of determination $\left(\mathrm{r}^{2}\right)$. The appearance viscosity $\left(\eta_{\mathrm{ap}}\right)$ of samples was determined by the average viscosity values of the viscosity curves.

After coacervation, samples were frozen in a biofreezer at $-20^{\circ} \mathrm{C}$ for $24 \mathrm{~h}$ and subsequently they were lyophilized in a L101 LIOTOP freezer-dryer for $12 \mathrm{~h}$. The structural and spectroscopic properties of the lyophilized sodium coacervates were studied by the following techniques:

Raman scattering spectra were recorded at room temperature in a frequency range of $200-1500 \mathrm{~cm}^{-1}$ in a HORIBA Jobin Yvon model LabRAM HR micro Raman apparatus equipped with a $632.8 \mathrm{~nm}$ laser delivering $17 \mathrm{~mW}$.

Differential scanning calorimetry (DSC) measurements for both $\mathrm{NaPC}$ lyophilized sodium coacervates and coacervate glass were carried out in a temperature range from 25 to $600{ }^{\circ} \mathrm{C}$ at a heating rate of $10{ }^{\circ} \mathrm{C} \mathrm{min}^{-1}$ using the DSC Q600 equipment from TA Instruments. In such conditions, samples were encapsulated in aluminum crucibles under a flowing nitrogen atmosphere (70 $\mathrm{mL} \mathrm{min}^{-1}$ ) and estimate error is $\pm 2{ }^{\circ} \mathrm{C}$ for $\mathrm{T}_{\mathrm{g}}$ and $\mathrm{T}_{\mathrm{x}}$.

Powder X-ray diffraction measurements were carried out with a Siemens Kristalloflex diffractometer operating with a Ni filtered $\mathrm{CuK} \alpha$ radiation source, step scanning was performed at $2 \theta$ angle ranging $10-80^{\circ}$ with a step pass of $0.01^{\circ}$ and a step time of $2 \mathrm{~s}$.

SEM images and energy dispersive X-ray spectroscopy (EDS) for the samples NaPC4.25 and NaPC30.8 were obtained using the field emission scanning electron microscope JEOL JSM-7500F.

Solid state NMR experiments were carried out at room temperature on a Bruker Avance III 400WB HD spectrometer, using a $4 \mathrm{~mm}$ MAS-NMR probe at a spinning speed of $14 \mathrm{kHz} .{ }^{31} \mathrm{P}$ NMR measurements were carried out at $162.0 \mathrm{MHz}$, using $90^{\circ}$ pulses of

Table 1

Compositions of the NaPC prepared by addition of different methanol molar fractions.

\begin{tabular}{lc}
\hline Samples & Methanol molar fractions $(x \%)$ \\
\hline NaPC4.25 & 4.25 \\
NaPC8.17 & 8.17 \\
NaPC11.8 & 11.8 \\
NaPC15.1 & 15.1 \\
NaPC18.2 & 18.2 \\
NaPC23.7 & 23.7 \\
NaPC30.8 & 30.8 \\
\hline
\end{tabular}


$3.2 \mu$ s length and a recycle delay of $300 \mathrm{~s}$. Chemical shifts were reported relative to $85 \% \mathrm{H}_{3} \mathrm{PO}_{4}$ with estimated error in \pm 0.1 . Signal deconvolutions into Gaussian components were done using the DMFIT software package with estimated error in $\pm 2 \%$ [24].

\section{Results and discussion}

\subsection{Coacervate characterization}

In general, the preparation of coacervate involves a sodium polyphosphate solution and a solution of two or three fold metallic ions like Ca, Co and Ni. Silva et al. and Dias Filho et al. studied the coacervation process of $\mathrm{Co}^{2+}, \mathrm{Ni}^{2+}$ and $\mathrm{Ca}^{2+}$ ions using $\mathrm{Na}\left(\mathrm{PO}_{3}\right)_{n}$ solution $[5,11,14]$. The first research studied the dynamical processes of occupancy sites in the coacervation process of the $\mathrm{Co}^{2+}$ and $\mathrm{Ni}^{2+}$ coacervates by X-ray Absorption Spectroscopy (EXAFS) and Raman Spectroscopy. In the second study, Dias Filho et al. showed the optical properties of sodium coacervate, containing $\mathrm{Ca}^{2+}$ ions, by using $\mathrm{Eu}^{3+}$ as a structural probe. It is important to emphasize, that the coacervation processes reported in the literature always occur in the exchange of $\mathrm{Na}^{+}$by cations such as $\mathrm{Co}^{2+}$, $\mathrm{Ni}^{2+}, \mathrm{Ca}^{2+}$ or other higher valence metallic ions. Although many studies on the scientific literature involve polyphosphate coacervate, up to now, there are no reports about formation of sodium coacervate.

In this paper, our methodology was based on the work proposed by Umegaki et al. [1]. These authors suggested that after the addition of methanol to sodium polyphosphate solution a liquidliquid phase separation occurs, considering the coacervate obtained as the denser phase [12,14]. In this paper, we studied the synthesis of sodium coacervates and how the addition of different amounts of methanol $(4.25 \leq x \leq 30.8 \% \mathrm{v} / \mathrm{v})$ to a fixed volume of $\mathrm{Na}\left(\mathrm{PO}_{3}\right)_{\mathrm{n}}$ solution $4.0 \mathrm{~mol} \mathrm{~L}-1$ can play in the structure of the coacervate.

Fig. 1 shows the liquid-liquid phase separation of the sodium coacervate (sample NaPC4.25). The coacervation process and liquid-liquid phase separation was observed for all samples described in Table 1.

In order to study the effect of increasing the amount of methanol on the properties of the coacervates, shear-dependent viscosities $(\eta)$ curves were obtained and are shown in Fig. 2.

The shear-dependent viscosities are shown in Fig. 2(a) for sodium polyphosphate coacervate at different volumes of methanol which revealed the Newtonian and non-Newtonian yield. In addition, shear-thinning was exhibited by NaPC8.17 sample. Shear

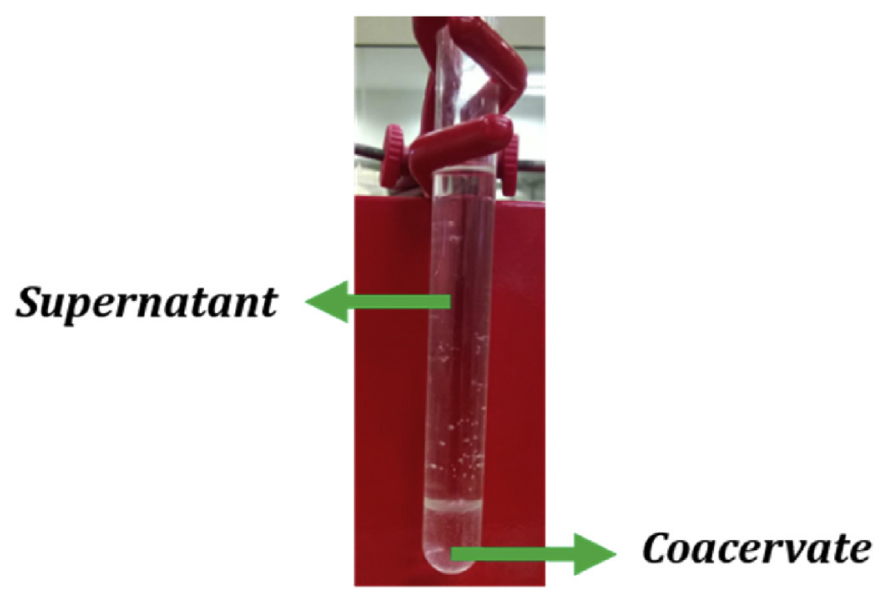

Fig. 1. Photography of the liquid-liquid phase separation for sample NaPC4.25. thinning was previously reported for other coacervates of polymers and proteins [25-27]. The graph reveals that a variation of solvent affected the viscosity of the system. The NaPC15.1, NaPC18.2, NaPC23.8 and NaPC30.8 samples exhibited a shear thickening. Shear thickening was previously reported for adhesive coacervates [28]. It is interesting to point out that the viscosity increased with increasing of methanol molar fraction. The viscosity of the coacervates could be directly related to the strength of the electrostatic or molecular interactions. However, the increasing viscosity is promising regarding the adhesion properties of these coacervates for future wet adhesive and coating technologies.

A power law was used to model the shear stress-shear rate data of samples, Fig. 2(b). Most samples exhibited Newtonian behavior $(\mathrm{n}=1)$, except NaPC11.8 sample that showed a pseudoplastic behavior $(\mathrm{n}<1)$. Samples NaPC18.2 and NaPC30.8 exhibited a dilatant behavior $(n>1)$ (Table 2$)$. That means the viscosity increases with increasing shear rate (dilatant flow) or the viscosity decreases with increasing shear rate (pseudoplastic flow). The consistency index $(\mathrm{k})$ increases by adding solvent indicating the increase of the viscosity of samples, which can be formed by a more structured network. The viscosity values at $100 \mathrm{~s}^{-1}$ corroborate to this fact, ie, the increase of the methanol molar fraction increases the viscosity of the coacervate. This can be explained by the formation of aggregates and/or by a further interaction.

In accordance with the Kraszewski equation, it is possible to estimate the dielectric constant for the water-methanol mixture at $20{ }^{\circ} \mathrm{C}$ as:

$\varepsilon_{\mathrm{m}}=\left(v_{1} \varepsilon_{1}^{\frac{1}{2}}+v_{2} \varepsilon_{2}^{\frac{1}{2}}\right)^{2}$

In this case, it is possible to see that the addition of methanol leads to a decrease of the dielectric mixture constant, where $\left(\varepsilon_{\mathrm{m}}\right)$ is the dielectric constant of binary mixture, $\left(\varepsilon_{1}\right)$ and $\left(\varepsilon_{2}\right)$ are the dielectric constant of water and methanol, respectively, $\left(v_{1}\right)$ is the water molar fraction and $\left(v_{2}\right)$ is the methanol molar fraction Fig. 3 [29].

As discussed above, the coacervation process for $\mathrm{Ni}^{2+}, \mathrm{Co}^{2+}$ and $\mathrm{Na}^{+}$coacervates occurs by reduction of the dielectric constant $(\varepsilon)$ of aqueous solution $\left(\varepsilon_{1}\right.$,water $\left.=80.4\right)$ by addition of a low molecular weight solvent, for example, methanol $\left(\varepsilon_{2, \text { methanol }}=32.3\right)$ $[1,2,4,5,30,31]$. In this sense, it is plausible to infer that the higher viscosity of NaPC30.8 can be linked to the presence of a higher amount of methanol and, consequently, with the reduction of the dielectric constant of the mixture, which can lead to approximation and subsequent better interactions between adjacent phosphate chains in solution, as represented in Fig. 4. Fig. 4 shows a proposal of the coacervation process for sodium coacervates during the addition of methanol. Before the addition of methanol, the polyphosphate chains are distant enough from each other due the presence of a higher and completely dissociated amount of water. After the addition of methanol and reduction of the dielectric constant, the approximation of the polyphosphates chains can take place leading to the coacervation process thanks to the destabilization of the aqueous system and the decreasing of the electrostatic repulsion between polyphosphate chains.

The addition of solvents, such as alcohols or acetone to aqueous sodium polyphosphate, in order to prepare coacervates is known through literature. However, in some cases, the addition of divalent cations occurs, as $\mathrm{Zn}^{2+}, \mathrm{Co}^{+}, \mathrm{Ni}^{2+}$ etc., for example, [5,12,14]. The novelty in this work is that the coacervation occurs by adding methanol to the polyphosphate without needing the presence of another cation. 

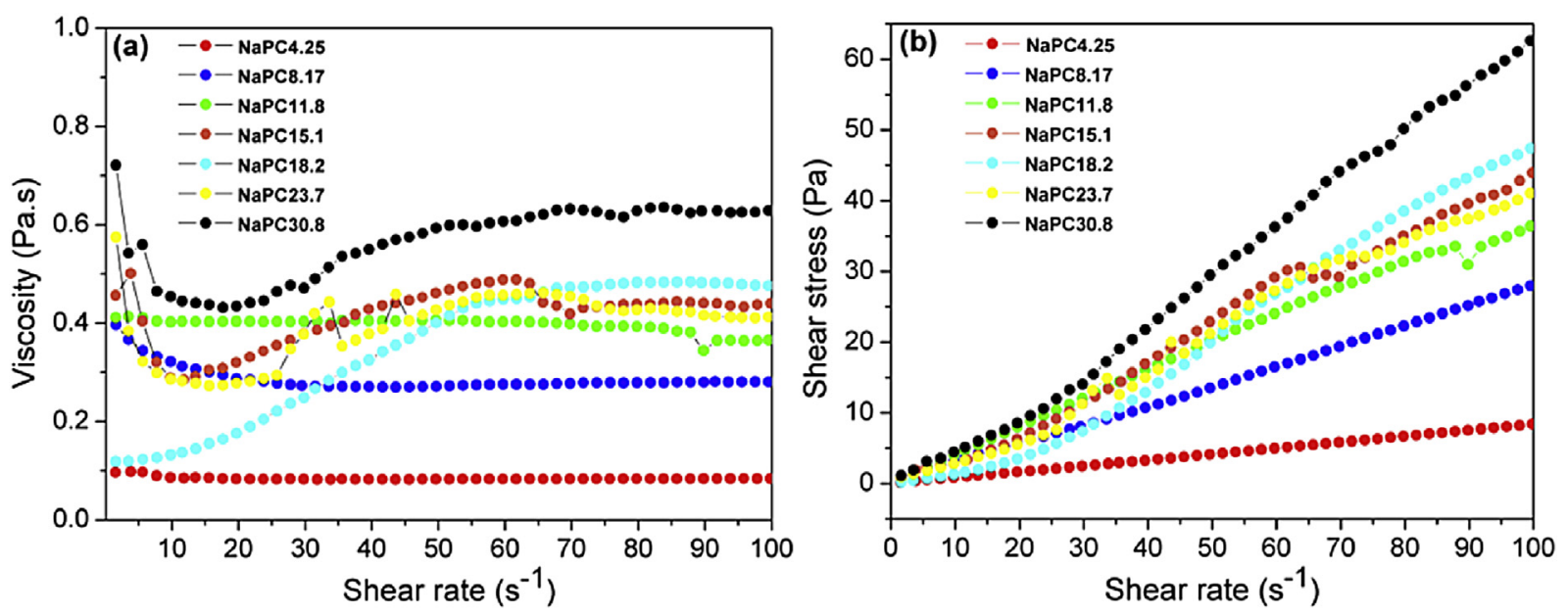

Fig. 2. (a) Viscosity as a function of shear rate (b) Shear stress as a function of shear rate for the NaPC samples.

Table 2

Parameters related to Power Law's model, appearance viscosity and viscosity at $100 \mathrm{~s}^{-1}$.

\begin{tabular}{lllllll}
\hline Sample & $\mathrm{k}$ & $\mathrm{n}$ & $\mathrm{r}^{2}$ & $\eta_{\text {ap }}($ Pa s $)$ & & $\eta\left(\right.$ Pa s) at $100 \mathrm{~s}^{-1}$ \\
\hline NaPC4.25 & 0.080 & 1.010 & 1.000 & 0.042218 & \pm 0.003 & 0.0841 \\
NaPC8.17 & 0.264 & 1.013 & 0.999 & 0.286424 & \pm 0.025 & 0.2808 \\
NaPC11.8 & 0.568 & 0.909 & 0.995 & 0.198376 & \pm 0.014 & 0.3656 \\
NaPC15.1 & 0.350 & 1.053 & 0.990 & 0.207531 & \pm 0.057 & 0.4761 \\
NaPC18.2 & 0.086 & 1.386 & 0.990 & 0.175275 & \pm 0.1360 & 0.4400 \\
NaPC23.7 & 0.326 & 1.061 & 0.985 & 0.197802 & \pm 0.067 & 0.4125 \\
NaPC30.8 & 0.310 & 1.159 & 0.998 & 0.282685 & \pm 0.0752 & 0.6291 \\
\hline
\end{tabular}

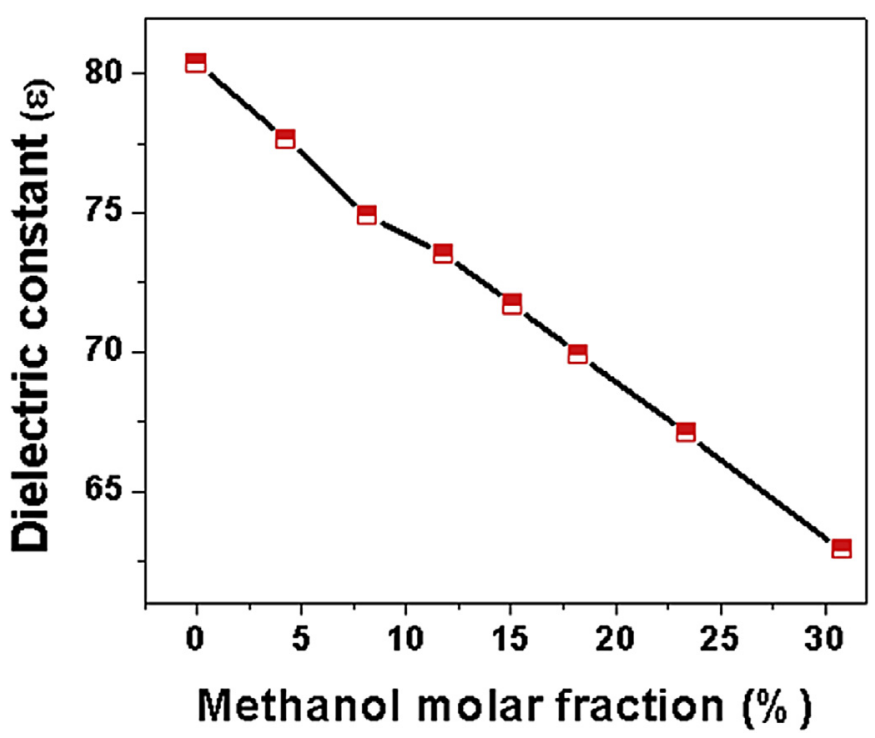

Fig. 3. Dielectric constant calculated by Kraszewski equation for water-methanol mixture as a function of the methanol molar fractions (\%) at $20^{\circ} \mathrm{C}$.

\subsection{Lyophilized sodium polyphosphate coacervates}

In order to evaluate the morphology and composition of the coacervates obtained, the NaPC4.25 lyophilized (the lowest methanol molar fraction) and NaPC30.8 lyophilized (the highest methanol molar fraction) were selected for the study and comparison by Field Emission Gun - Scanning Electron Microscope/Energy
Dispersive Spectroscopy (FEG- SEM/EDS) analysis.

Two representatives SEM images of coacervate samples are shown in Fig. 5(a) and (b). They illustrate the morphology of the $\mathrm{NaPC}$ samples. SEM images show the fractures on the surface of both materials after the drying process.

EDS spectra showed that both coacervates purely present $\mathrm{Na}, \mathrm{P}$ and $\mathrm{O}$ elements and have almost the same $\mathrm{Na} / \mathrm{P}$ ratio, as can be seen in Fig. 5(c) and (d). The Na/P ratio for the NaPC4.25 and NaPC30.8 are 1.11 and 1.24 , respectively. The result shows that after the addition of different methanol molar fractions, the values of the $\mathrm{Na}$ / $P$ ratio do not change considerably.

\subsubsection{Structural characterization}

The structures of the lyophilized coacervates have been investigated by solid-state ${ }^{31} \mathrm{P}$ MAS-NMR. The $\mathrm{Q}^{\mathrm{n}}$ notation, in which $\mathrm{n}=0,1,2,3$ represents the number of bridging oxygens of phosphate group [32]. The $Q^{n}$ connectivity change of phosphate groups has been studied by NMR, using the ${ }^{31} \mathrm{P}$ nuclei as structural probe for a set of glass compositons of simple alkali and alkaline earth glasses [33,34] as well as for other systems [35].

The ${ }^{31} \mathrm{P}$ MAS-NMR spectra of sodium coacervates for NaPC samples are showed in Fig. 6. The ${ }^{31}$ P MAS-NMR spectra show three resonance lines: centered at $3.0 \pm 0.1,-6.7 \pm 0.1$ and $-19.2 \pm 0.1 \mathrm{ppm}$. They are attributed to $\mathrm{Q}^{0}, \mathrm{Q}^{1}$ and $\mathrm{Q}^{2}$ units, respectively. This results show that the lyophilized coacervate are still present in a small fraction of isolated $\left(\mathrm{Q}^{0}\right)$ phosphate units, as observed in Graham salt. Table 3 summarizes the percentages of $\mathrm{Q}^{0}$, $\mathrm{Q}^{1}$ and $\mathrm{Q}^{2}$ units in the sodium coacervate samples and for the Graham salt.

The data obtained from the integration of the spectra show that the lyophilized coacervates maintain the same concentrations of $Q^{0}$ units regardless of the methanol concentration. However, the data also shows that the addition of methanol leads to a decrease of $\mathrm{Q}^{2}$ units and consequently the $\mathrm{Q}^{1}$ units increase, when compared to Graham salt. The polyphosphate chains are formed by $\mathrm{Q}^{1}$ terminal phosphate units and $\mathrm{Q}^{2}$ m phosphate middle chain units. Thus the average size of the chain can be represented as $w=\left(Q^{2} m\right)+2 Q^{1}$, in which $m$ is the number of units in presented in the chain $Q^{2}$. This result suggests that the coacervation process of small polyphosphate chains, such as pyrophosphate and tripolyphosphate, is not stabilized inside the coacervate, therefore remaining in the supernatant, i.e., the coacervate is formed only from long chains and, in this case, a reduction in dielectric constant solution leads to a hydrolysis of the chains. 


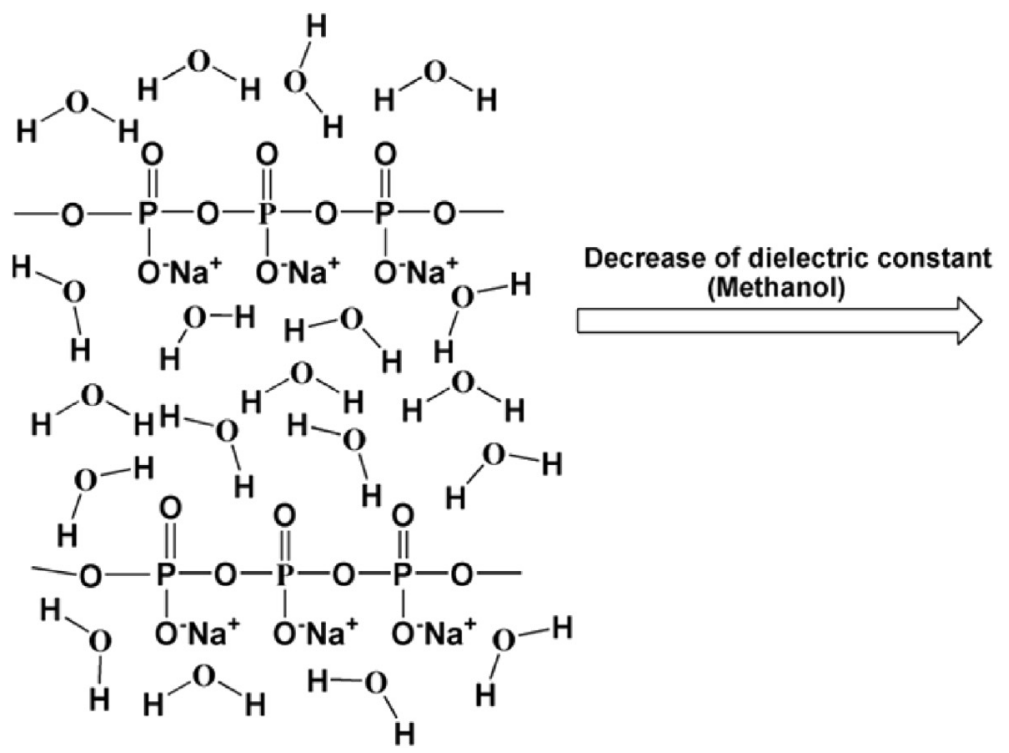

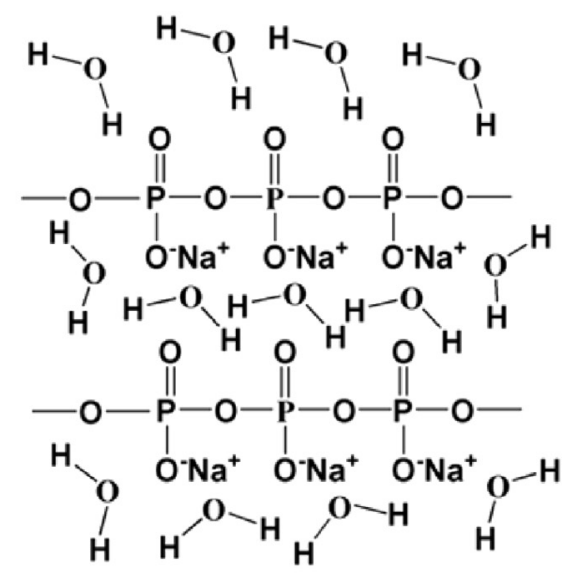

Fig. 4. Schematic representation of coacervation process for NaPC after the addition of methanol. Decreasing the dielectric constant $(\varepsilon)$ allows the approximation of polyphosphate chains and a denser phase.
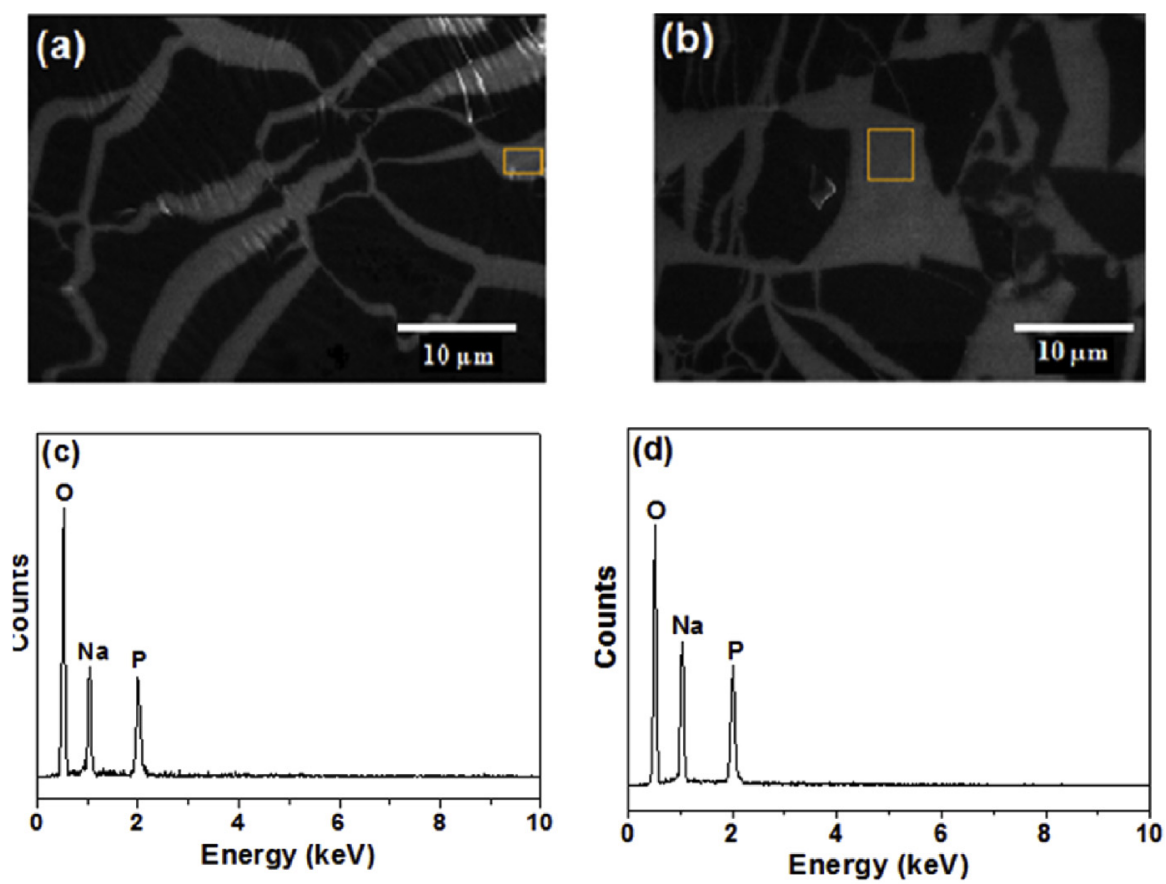

Fig. 5. SEM images for the samples NaPC4.25 (a) and NaPC30.8 (b) EDS spectra of the same samples NaPC4.25 (c) and NaPC30.8 (d).

Fig. 7 shows the Raman spectra for all samples of sodium coacervate obtained using a $632.8 \mathrm{~nm}$ laser excitation. As can be seen, the two most intense bands falls in, approximately, 680 and $1160 \mathrm{~cm}^{-1}$. Such bands can be assigned to $\mathrm{P}-\mathrm{O}_{\mathrm{b}}$ (bridged) and $\mathrm{P}-\mathrm{O}_{\mathrm{t}}$ (terminal) symmetric stretching modes, respectively [5,14].

The band at $1163 \mathrm{~cm}^{-1}$, observed in the Raman spectrum of Graham salt, shifts slightly to $1160 \mathrm{~cm}^{-1}$ after the addition of methanol. The shift can be explained by the higher sensitivity of the terminal groups $\left(\mathrm{P}-\mathrm{O}_{\mathrm{t}}\right)$ to electrostatic forces present in the coacervate phase, due to increase of interaction between adjacent polyphosphate chains and the rise in the methanol concentration. As discussed above, the reduction of the dielectric constant by addition of different methanol molar fractions promotes the approximation of the bordering chains polyphosphates due to the presence of the terminal groups $\left(\mathrm{P}-\mathrm{O}_{\mathrm{t}}\right)$.

Fig. 8 shows a shift on the vibrational modes $u_{s}\left(\mathrm{P}-\mathrm{O}_{\mathrm{b}}\right)$ and $u_{s}(\mathrm{P}-$ $\mathrm{O}_{t}$ ) as a function of the methanol molar fraction. In this case, as can be observed, the $u_{s}\left(P-O_{b}\right)$ vibrational mode does not change considerably as a function to increase of the molar fraction of methanol in the sodium polyphosphate solution. This observation corroborates to the ${ }^{31} \mathrm{P}$ NMR results obtained for the coacervates. On the other hand, the vibrational mode $v_{s}\left(\mathrm{P}_{-} \mathrm{O}_{t}\right)$ is more sensitive to the reduction of the dielectric constant and shifts due to the proximity to other polyphosphate chains, as showed in Fig. 4. 


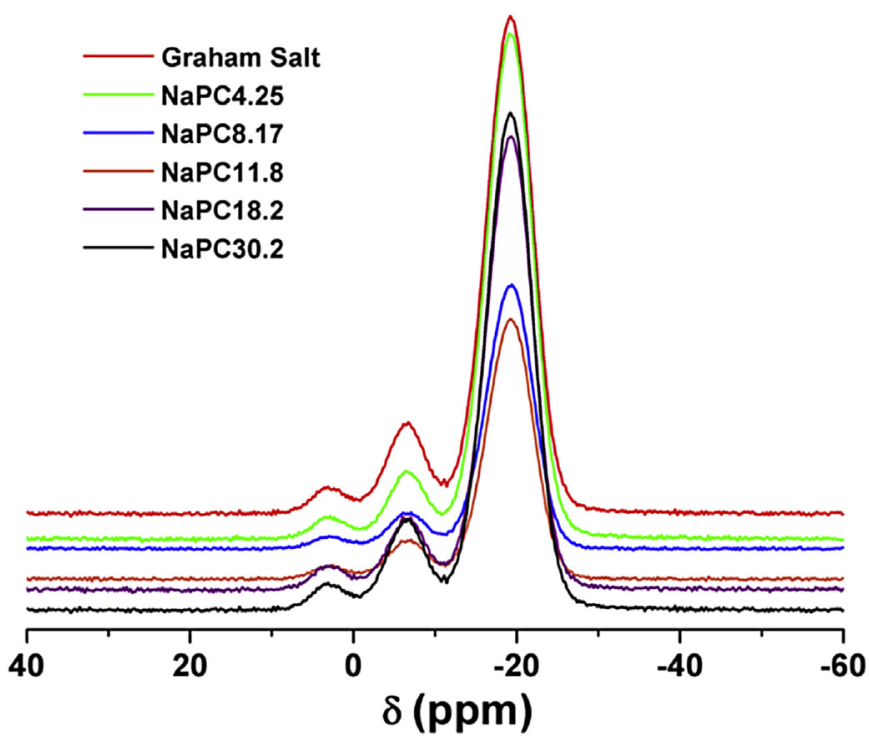

Fig. 6. ${ }^{31} \mathrm{P}$ NMR spectra of the NaPC samples with different methanol molar fractions and the precursor $\mathrm{Na}\left(\mathrm{PO}_{3}\right)_{n}$.

The structural evolution of the coacervation process as a function of the methanol molar fraction can be discussed regarding both ratio $Q^{2} / Q^{1}$ units and $A_{\cup_{S}\left(\mathrm{P}-O_{f}\right)} / A_{\cup_{S}\left(\mathrm{P}-O_{\mathrm{b}}\right)}$, which were calculated by integrating the area (A) of ${ }^{31} \mathrm{P}$ MAS-NMR and Raman data, respectively [36,37]. Fig. 9(a) and (b) show the decrease of both $A_{v_{S}\left(P-O_{t}\right)} / A_{v_{S}\left(P-O_{b}\right)}$ and $Q^{2} / Q^{1}$ ratios, suggesting that the methanol favors hydrolysis of $\mathrm{P}-\mathrm{O}-\mathrm{P}$ bond in the middle of the chains and thus forming smaller polyphosphate chains. The model of coacervation process is in agreement with the data observed from the NMR and Raman data, in which they clearly show the influence concerning the vibrational mode of $\mathrm{P}-\mathrm{O}-\mathrm{P}$ bonds and variation of the $Q^{2} / Q^{1}$ ratio. As discussed above in the results of rheological measurements, the pseudoplastic behavior may be linked to the decrease of the $Q^{2}$ groups and changes in the chain conformations. The reduction of the viscosity in low shear rate of the some sodium coacervates is directly linked to structural change of the polymer chains.

\subsection{Coacervates glasses polyphosphate}

Polyphosphate coacervates are good precursors for preparation of glassy materials. Depending on the synthesis conditions, the coacervate can be molded into a transparent material at room temperature. However, it does not means that it will be a glassy material. In this work, we dried the coacervate at room temperature and studied it by means of DSC and X-ray diffraction.

As expected, despite it presents an amorphous character in the $\mathrm{X}$-ray diffraction, as shown in Fig. 10 it did not show the presence of glass transition in the DSC curve (Fig. 10(b)) and, for this reason, it can not be assumed as a glass.

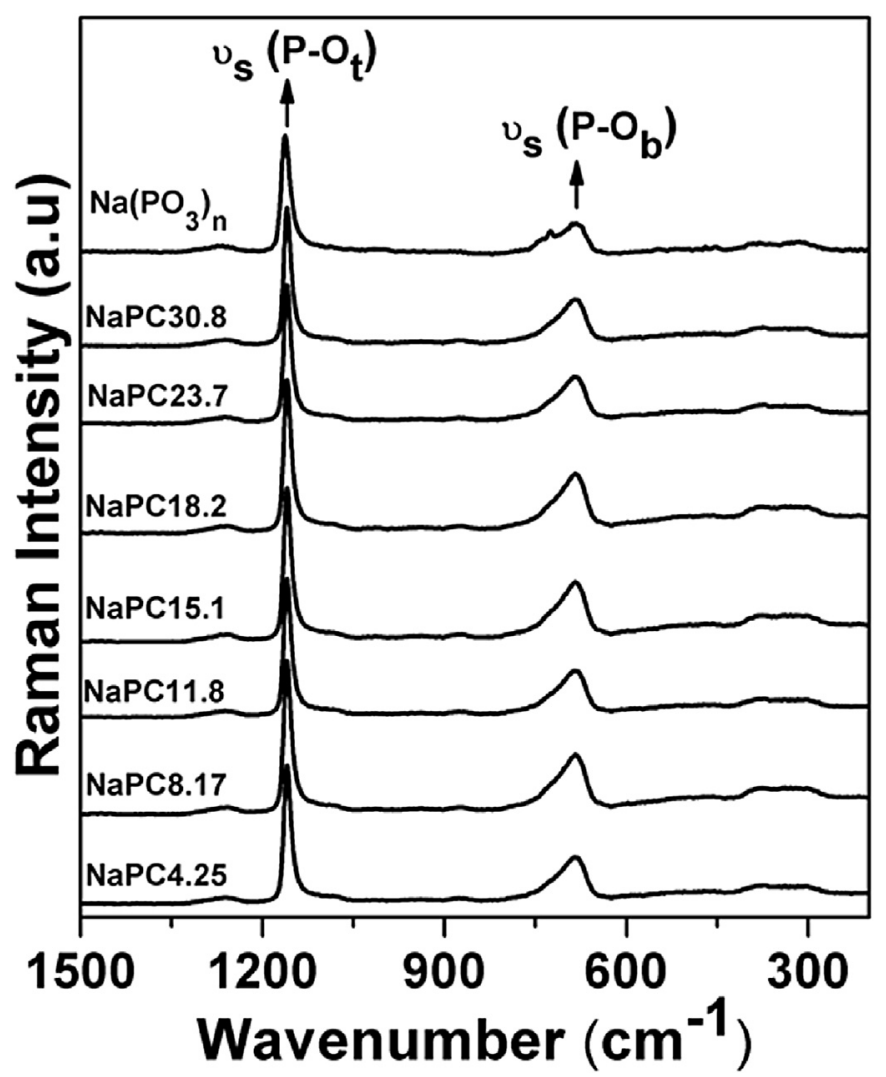

Fig. 7. Raman spectra of $\mathrm{NaPC}$ with different methanol molar fractions and $\mathrm{Na}\left(\mathrm{PO}_{3}\right)_{\mathrm{n}}$ obtained with $632.8 \mathrm{~nm}$ laser excitation.

Fig. 10(a) shows the amorphous X-ray diffraction pattern obtained from the sample NaPC4.24. This material was maintained for five days at room temperature in a vacuum desiccator containing silica-gel. In the inset, it is possible to see that the material is transparent.

Fig. 10(b) shows the DSC of the NaPC4.24 sample and it is possible to see three main events at 132,175 and $348{ }^{\circ} \mathrm{C}$. The two first events are assigned to phosphate chains crystallization, while the third one corresponds to a phase change process from orthophosphate to polyphosphates and/or pyrophosphates species [38]. As discussed above, no glass transition event was observed.

According to the results of solid-state ${ }^{31} \mathrm{P}$ MAS-NMR and Raman Scattering, the sample NaPC4.25 showed $\mathrm{Q}^{2} / \mathrm{Q}^{1}$ and $\mathrm{A}_{\mathrm{us}(\mathrm{P}-\mathrm{Ot})} / \mathrm{A}_{\mathrm{us}(\mathrm{P}-}$ ob) ratios higher than all samples analyzed, with is consistent with the presence of longer polyphosphate chains. Such phosphates are more suitable to prepare glasses than polyphosphate with smaller chains. Therefore, we decided to show the potential of this sample as a precursor for optical materials. Actually, new studies are in progress in our research group focusing on the influence of the different solvents on the coacervation process and glasses

Table 3

Integrated area ratio (\%) obtained in sodium lyophilized coacervates and Graham salt samples obtained from ${ }^{31} \mathrm{P}$ NMR data.

\begin{tabular}{|c|c|c|c|c|}
\hline Methanol molar fractions (\%) & $\mathrm{Q}^{0}($ area \%) & $\mathrm{Q}^{1}($ area $\%)$ & $\mathrm{Q}^{2}$ (area \%) & Chain size \\
\hline 0 & $2 \pm 2$ & $14 \pm 2$ & $84 \pm 2$ & 14 \\
\hline 4.25 & $2 \pm 2$ & $9 \pm 2$ & $89 \pm 2$ & 22 \\
\hline 8.17 & $2 \pm 2$ & $10 \pm 2$ & $88 \pm 2$ & 19 \\
\hline 11.8 & $2 \pm 2$ & $11 \pm 2$ & $87 \pm 2$ & 18 \\
\hline 18.2 & $2 \pm 2$ & $11 \pm 2$ & $87 \pm 2$ & 18 \\
\hline 30.8 & $2 \pm 2$ & $12 \pm 2$ & $86 \pm 2$ & 15 \\
\hline
\end{tabular}




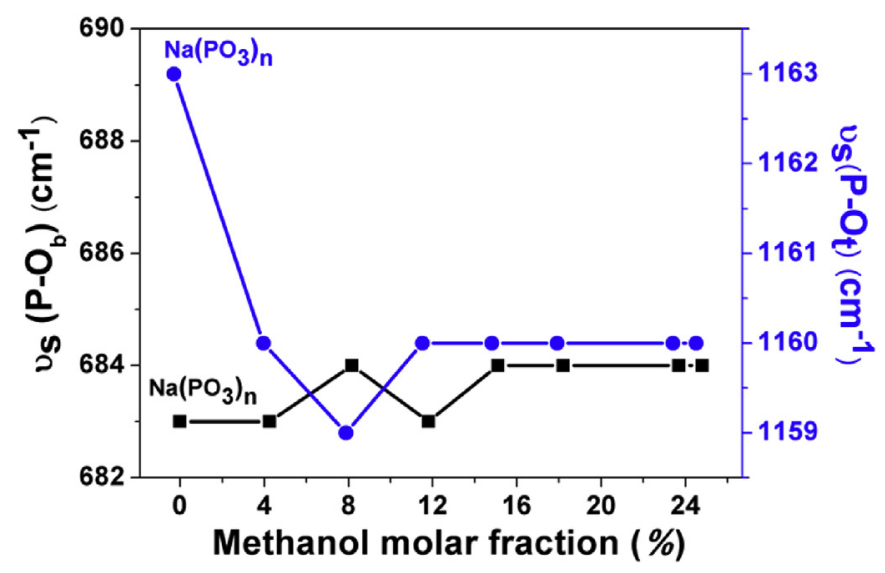

Fig. 8. Dependence of the wavenumber for both vibrational modes of NaPC: $\cup_{s}\left(\mathrm{P}-\mathrm{O}_{b}\right)$ (square) and $v_{s}\left(\mathrm{P}-\mathrm{O}_{\mathrm{t}}\right)$ (circle).

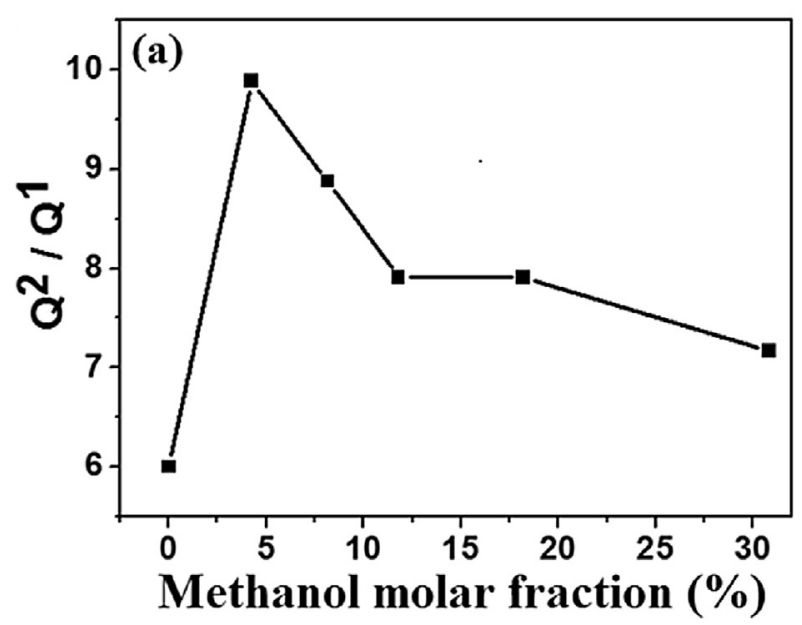

preparation.

Concerning to the preparation of the sodium coacervate glass using the same composition (NaPC4.24), the material was lyophilized, grinded in agate mortar and melted in platinum crucible at $800{ }^{\circ} \mathrm{C}$ for $10 \mathrm{~min}$, under atmospheric conditions. The melting was quenched between two steel plates resulting in a transparent material, as showed in the inset of Figure 11 beside the DSC curve of the sodium coacervate glass. Different from the case described above, the DSC curve exhibits a glass transition event at $271{ }^{\circ} \mathrm{C}$ and an exothermic peak in $351{ }^{\circ} \mathrm{C}$, assigned to crystallization process, therefore confirming the formation of a glassy material. Despite to be a single component glass, the glass stability parameter is considerably high $\left(\Delta \mathrm{T}=\mathrm{T}_{\mathrm{x}}-\mathrm{T}_{\mathrm{g}}=80^{\circ} \mathrm{C}\right)$. On the other hand, as known for high concentration polyphosphates glasses, it is hygroscopic. In order to improve the chemical material resistance, another chemical component, such as $\mathrm{ZnO}$, must be added.

\section{Conclusion}

Sodium Polyphosphates Coacervates (NaPC) were prepared through direct addition of different methanol molar fractions to a

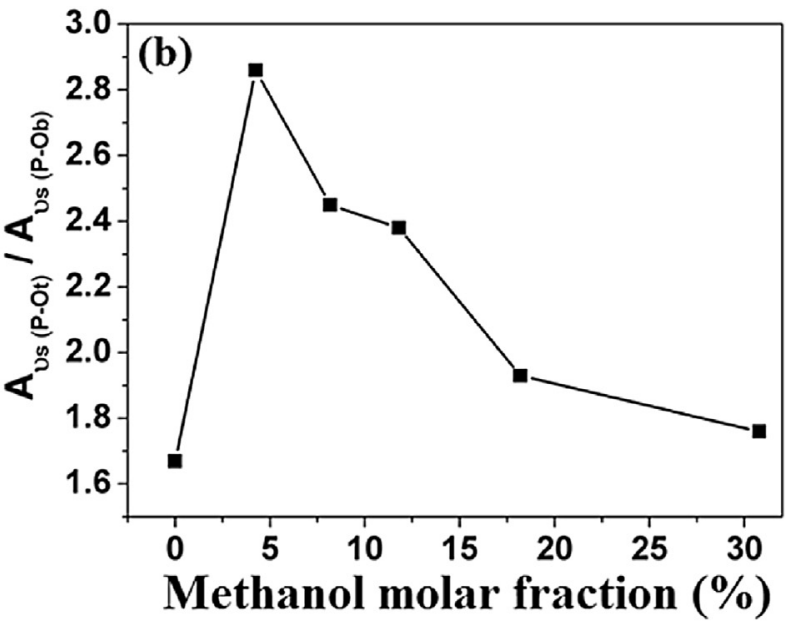

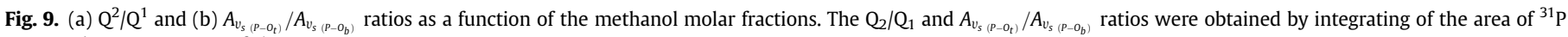
NMR and Raman spectra of the NaPC.
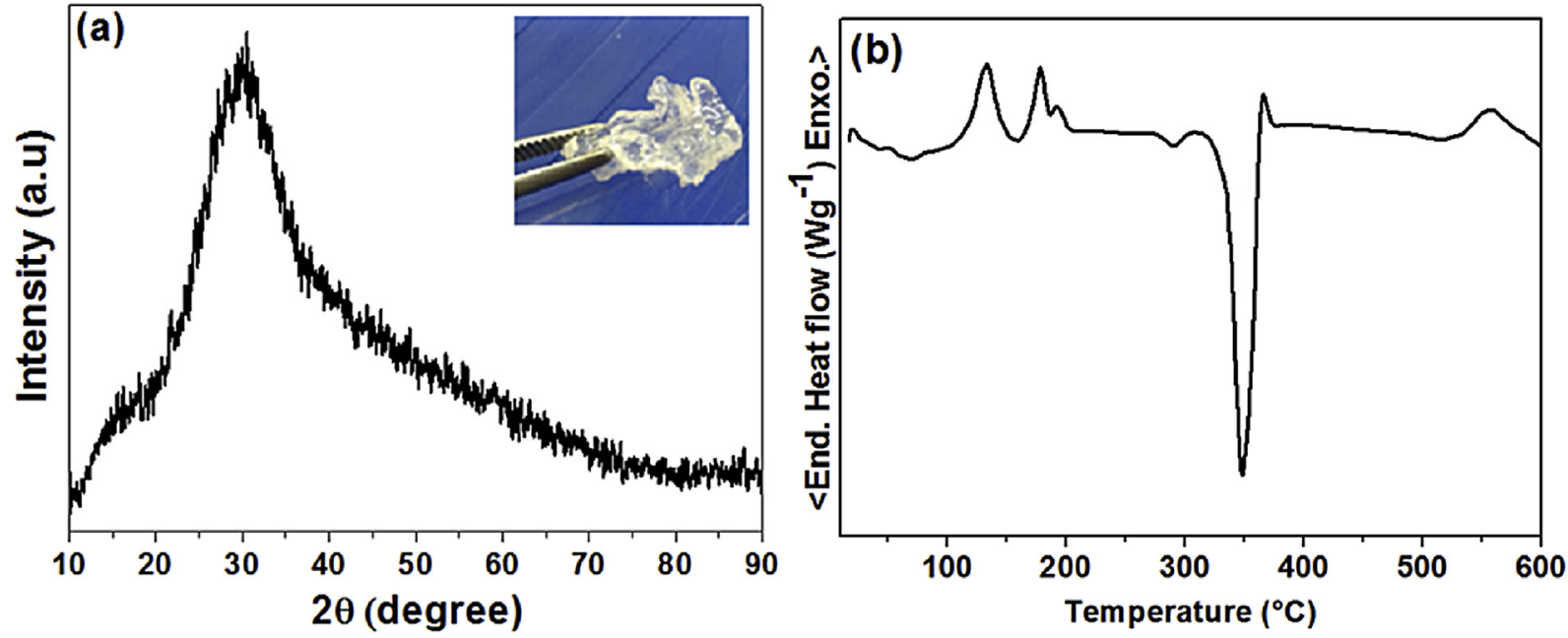

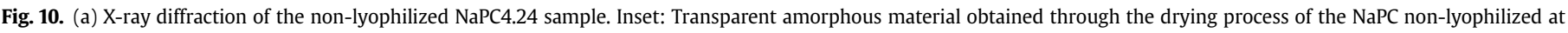
room temperature. (b) DSC curve of the non-lyophilized NaPC4.24 sample. 


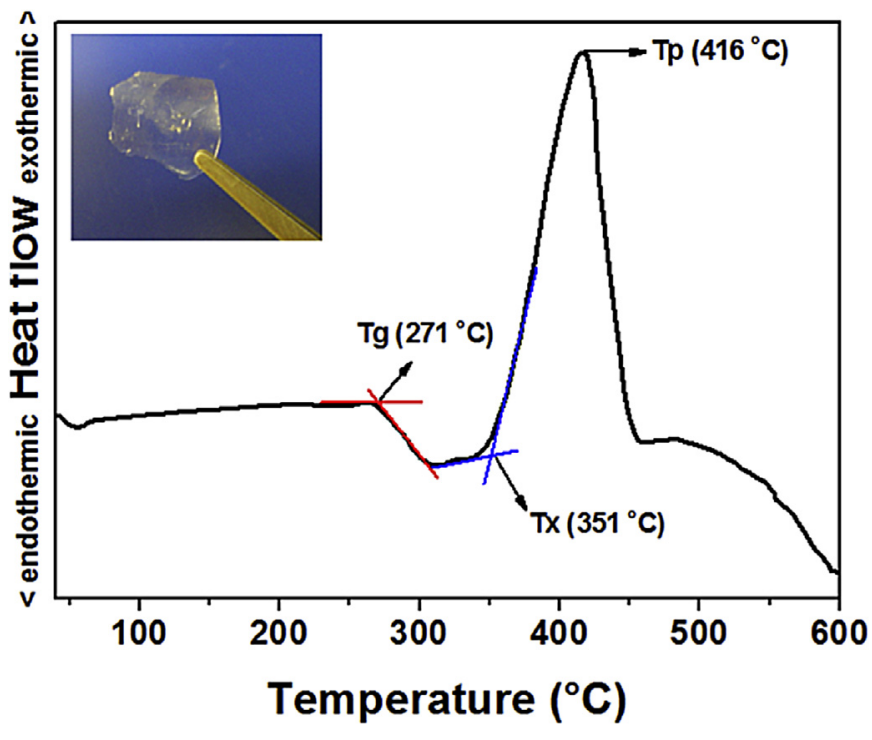

Fig. 11. DSC curve of the NaPC4.24 glass. Inset: Image of the NaPC glass.

sodium polyphosphate solution. The coacervation process and liquid-liquid phase separation was observed on all samples prepared without the need to add others metallic ions. The rheology measurements showed the viscosity increase of NaPC with the addition of methanol and most NaPC samples exhibited Newtonian behavior. The calculation of the dielectric constant of watermethanol system showed a decrease on these values with an increase of methanol molar fractions. The structural characterization by solid-state ${ }^{31} \mathrm{P}$ MAS-NMR and Raman Scattering showed that the addition of methanol causes a decrease of $\mathrm{Q}^{2} / \mathrm{Q}^{1}$ species and $A_{v_{S}\left(P-O_{t}\right)} / A_{v_{S\left(P-O_{b}\right)}}$ indicating the hydrolysis of the polyphosphate chains. On the other hand, NaPC can be used as soft Glass-Materials precursors.

\section{Acknowledgments}

Financial support of the Brazilian agencies Capes/PNPD grant \# 2654/2011 and grant \# 2013/07793-6 São Paulo Research Foundation FAPESP/CEPID are gratefully acknowledged. The authors would like to thanks Prof. C. V. Santilli from Chemistry Institute of UNESP for the rheological measurements.

\section{References}

[1] T. Umegaki, Y. Nakayama, T. Kanazawa, Thermal change of magnesium high polyphosphate coacervates, Bull. Chem. Soc. Jpn. 49 (1976) 2105-2107.

[2] H.G. Bungenberg De Jong, H. Kruyt, Concentration of matter and action of enzymes on coacervate, Kolloid Z 50 (1930) 39.

[3] H.G. Bungenberg de Jong, Crystallization, coacervation and flocculation, Colloid Sci. 1 (1949) 232.

[4] F.M. Menger, B.M. Sykes, Anatomy of a coacervate, Langmuir 14 (1998) 4131-4137.

[5] M.A.P. Silva, D.F. Franco, A.R. Brandão, H.S. Barud, F.A. Dias Filho, S.J.L. Ribeiro, Y. Messaddeq, L.F.C. De Oliveira, Spectroscopic studies on glassy Ni(II) and Co(II) polyphosphate coacervates, Mater. Chem. Phys. 124 (2010) 547-551.

[6] T. Umegaki, T. Kanazawa, Viscosity behavior of coacervates of magnesium and calcium highpolyphosphates, Bull. Chem. Soc. Jpn. 48 (1975) 1452-1454.

[7] L.F.C. De Oliveira, M.A.P. Silva, A.R. Brandão, R. Stephani, C.I.R. De Oliveira, R.R. Gonçalves, A.J. Barbosa, H.S. Barud, Y. Messaddeq, S.J.L. Ribeiro, Amorphous manganese polyphosphates: preparation, characterization and incorporation of azo dyes, J. Sol-Gel Sci. Technol. 50 (2009) 158-163.

[8] N.C. Masson, E.F. De Souza, F. Galembeck, Calcium and iron (III) polyphosphate gel formation and aging, Colloids Surfaces A Phys. Eng. Aspects 121 (1997) $247-255$.

[9] E.C.O. Lima, J.M.M. Neto, Y.F. Fujiwara, F. Galembeck, Aluminum polyphosphate thermoreversible gels: a study by ${ }^{31} \mathrm{P}$ and ${ }^{27} \mathrm{Al}$ NMR spectroscopy, J. Colloid Interface Sci. 176 (1995) 388-396.
[10] A. Momeni, M.J. Filiaggi, Comprehensive study of the chelation and coacervation of alkaline earth metals in the presence of sodium polyphosphate solution, Langmuir 30 (2014) 5226-5266.

[11] F.A. Dias Filho, L.D. Carlos, Y. Messaddeq, S.J.L. Ribeiro, Spectroscopic study and local coordination of polyphosphate colloidal systems, Langmuir 21 (2005) 1776-1783.

[12] G. Willot, F. Gomez, P. Vast, V. Andries, M. Martines, Y. Messaddeq, M. Poulain, Preparation of zinc sodium polyphosphates glasses from coacervates precursors, Characterisation of the obtained glasses, and their applications, C. R. Chim. 5 (2002) 899-906.

[13] J.M. Casas, M.P. Garcia, M. Sanz, F. Cacho, J. Pérez, ${ }^{31} \mathrm{P}$ NMR spectroscopic studies of the influence of the environment in the degradation process of the Graham's salt, Ceram. Int. 36 (2010) 39-46.

[14] M.A.P. Silva, D.F. Franco, L.F.C. De Oliveira, New insight on the structural trends of polyphosphate coacervation processes, J. Phys. Chem. A 112 (2008) 5385-5389.

[15] K. Wang, F. Chen, C. Liu, C. Rüssel, The effect of polymeric chain-like structure on the degradation and cellular biocompatibility of calcium polyphosphate, Mater. Sci. Eng. C 28 (2008) 1572-1578.

[16] Y.L. Ding, Y.W. Chen, Y.J. Qin, G.Q. Shi, X.X. Yu, C.X. Wan, Effect of polymerization degree of calcium polyphosphate on its microstructure and in vitro degradation performance, J. Mater. Sci. Mater. Med. 19 (2008) 1291-1295.

[17] C.I.R. De Oliveira, L.F.C. De Oliveira, F.A. Dias Filho, Spectroscopic investigation of a new hybrid glass formed by the interaction between croconate ion and calcium polyphosphate, Spectrochim. Acta. Part A 61 (2005) 2023-2028.

[18] A. Dion, G. Hall, M.J. Filiaggi, The effect of processing on the structural characteristics of vancomycin-loaded amorphous calcium phosphate matrices, Biomaterials 26 (2005) 4486-4494.

[19] D.M. Pikup, R.J. Newport, E.R. Barney, J. Kim, S.P. Valappil, J.C. Knowles, Characterisation of phosphate coacervates for potential biomedical applications, J. Biomater. Appl. 28 (2014) 1226-1234.

[20] F.A. Dias Filho, S.J.L. Ribeiro, R.R. Gonçalves, Y. Messaddeq, L.D. Carlos, V. De Zea Bermudez, J. Rocha, $\mathrm{Eu}^{3+}$ doped polyphosphate-aminosilane organic-inorganic hybrids, J. Alloy. Compd. 374 (2004) 74-79.

[21] Q.Y. Ma, S.J. Traina, T.J. Logan, J.A. Ryan, Effects of aqueous Al, Cd, Cu, Fe(II), Ni, and $\mathrm{Zn}$ on $\mathrm{Pb}$ immobilization by hydroxyapatite, Environ. Sci. Technol. 28 (1994) 1219.

[22] K. Saegusa, F. Ishii, Triangular phase diagrams in the phospholipid-wateralcohol system for the preparation of lipid vesicles (liposomes) using the coacervation technique, Langmuir 18 (2002) 5984-5988.

[23] A. Momeni, M.J. Filiaggui, Rheology of polyphosphate coacervates, Soc. Rheol. 60 (2016) 25-34.

[24] D. Massiot, F. Fayon, M. Capron, I. King, S. Le Calve, B. Alonso, B.B. Durand, Z. Gan, G. Hoatson, Modelling one- and two-imensional solid-state NMR spectra, Magn. Reson. Chem. 40 (2002) 70-76.

[25] A.B. Kayitmazer, S.P. Strand, C. Tribet, W. Jaeger, P.L. Dubin, Effect of polyelectrolyte strusturee on protein-polyelectrolyte coacervates: coacervates of bovine serum albumin with poly(diallyldimethylammonium chloride) versus chitosan, Biomacromolecules 11 (2007) 3568-3577.

[26] H. Bohidar, P.L. Dubin, P.R. Majhi, C. Tribet, W. Jaeger, Effects of proteinpolyelectrolyte affinity and polyelectrolyte molecular weight on dynamic properties of bovine serum albumin-poly(diallyldimethylammonium chloride) coacervates, Biomacromolecules 6 (2005) 1573-1585.

[27] S.G. Gorji, E.G. Gorji, M.A. Mohammadifar, A. Zargaraan, Complexation of sodium caseinate with gum tragacanth: effect of various species and rheology of coacervates, Int. J. Biol. Macromol. 67 (2014) 503-511.

[28] D.S. Hwang, H. Zeng, A. Srivastava, D.V. Krogstad, M. Tirrell, J.N. Israelachvili, J.H. Waite, Viscosity and interfacial properties in a mussel-inspired adhesive coacervate, Soft Matter 6 (2010) 3232-3236.

[29] A. Megriche, A. Belhadj, A. Mgaidi, Microwave dielectric properties of binary solvent water- alcohol, alcohol-alcohol mixtures at temperatures between -35 ${ }^{\circ} \mathrm{C}$ and $+35{ }^{\circ} \mathrm{C}$ and dielectric relaxation studies, Mediterr. J. Chem. 1 (2012) 200-209.

[30] G. Palavit, L. Montagne, R. Delaval, Preparation of zinco-sodium phosphate glass precursors by coacervation, J. Non-Cryst. Solids 1893 (1995) 277-282.

[31] G. Åkerlöf, Dielectric constants some organic solvente-water mixtures at various temperatures, J. Am. Chem. Soc. 54 (1932) 4125-4139.

[32] T.M. Alam, R.K. Brow, Local structure and connectivity in lithium phosphate glasses: a solid-state 31P MAS NMR and 2D exchange investigation, J. NonCryst. Solids 223 (1998) 1-20.

[33] R.K. Brow, Review: the structure of simple phosphate glasses, J. Non-Cryst. Solids 263\&264 (2000) 1-28.

[34] S. Prabakar, K.T. Mueller, Solid-state NMR investigations of sodium-cesium mixed-alkali phosphate glasses, J. Non-Cryst. Solids 349 (2004) 80-87.

[35] A. Angelopoulou, V. Montouillout, D. Massiot, G. Kordas, Study of the alkaline environment in mixed alkali compositions by multiple-quantum magic angle nuclear magnetic resonance (MQ-MAS NMR), J. Non-Cryst. Solids 354 (2008) $333-340$.

[36] J.E. Pemberton, L. Latifzadeh, Raman spectroscopy of calcium phopshate glasses with varying $\mathrm{CaO}$ modifier concentrations, Chem. Mater. 3 (1991) 195-200.

[37] J. Koo, B. Bae, H. Na, Raman spectroscopy of copper phosphate glasses, J. NonCryst. Solids (1997) 173-179.

[38] T. Umegaki, Y. Nakayama, T. Kanazawa, Thermal change of magnesium highpolyphosphate coacervate, Bull. Chem. Soc. Jpn. 49 (1976) 2105-2107. 